\title{
Choosing the function of mechanical properties of grounds and rock formations due to their heterogeneity
}

\author{
Irina Frolova ${ }^{1, *}$ and Murad Agakhanov ${ }^{1}$ \\ ${ }^{1}$ Moscow State University of Civil Engineering, Yaroslavskoye sh., 26, 129337 Moscow, Russia
}

\begin{abstract}
The development of computing techniques to analyze underground structures, buildings in high-rise construction that would fully take account of the conditions of their design and operation, as well as the real material properties, is one of the important trends in structural mechanics. For the territory in high-rise construction it is necessary to monitor the deformations of the soil surface. When high-rise construction is recommended to take into account the rheological properties and temperature deformations of the soil, the effect of temperature on the mechanical characteristics of the surrounding massif. Similar tasks also arise in the creation and operation of underground parts of high-rise construction, which are used for various purposes. These parts of the structures are surrounded by rock massifs of various materials. The actual mechanical characteristics of such materials must be taken into account. The objective property of nearly all materials is their non-homogeneity, both natural and technological. The work addresses the matters of building nonhomogeneous media initial models based on the experimental evidence. This made it possible to approximate real dependencies and obtain the appropriate functions in a simple and convenient way.
\end{abstract}

\section{Introduction}

Media with mechanical properties continuous nonhomogeneity by its description can be conventionally divided into two groups to be named as media with direct and indirect heterogeneity. The first one is presented by the materials with mechanical properties that obtained various values in terms of a volume of a body under consideration during the period of manufacturing (the cementation process of the rock mass that surrounds the underground cavity, explosion loads etc.) The second group comprises materials with properties that vary during operation (temperature, radiation effects etc.) When selecting functions that describe properties change alongside the coordinates of a body from material falling into the first group, only the results of experimental investigations should be based

\footnotetext{
*Corresponding author: ifrolova51@inbox.ru
} 
on, hence one can approximate real dependencies so that the functions obtained are relatively simple and still represent the facts.

In problems with indirect inhomogeneity the change of mechanical characteristics alongside the body was brought about not only by the dependence of properties upon a trigger (temperature, radiation) but upon distribution of this trigger in the body as well. The first part of the problem can be solved similarly as in the bodies with direct inhomogeneity, while the second part results from some equations solution, for instance, a heat conduction equation in thermoelasticity problems.

The selection of mechanical characteristics variation law in rock formations and grounds is base on ideas of rocks and grounds mechanics as well as experimental research.

The current investigations in the Earth's physics [1], rock mechanics [2] and ground mechanics [3] the natural (earthy) non-homogeneity is characterized by a medium modulus of elasticity variation by depth. Virgin ground modulus of elasticity is represented by means of exponential function of depth $[1,3]$.

Along with this, the construction of mine workings (underground tanks) means an artificial change in the mechanical characteristics of the surrounding massif. The massif acquires the so-called technological heterogeneity due to the penetration of the mine. Small violations of physical continuity arise on the contour of the cavity and rapidly decay with fading into the interior of the massif.

As the results of existing experimental studies show [4], the resulting fracturing leads to a decrease in the modulus of elasticity. Near the hole, where the fracture is more intense, the elastic modulus is small, and as it moves away from the cavity contour into the interior of the massif, it grows asymptotically tending to the value existing before cavity penetration (Fig. 1).

In this case, the modulus of elasticity of a weighted medium with a spherical cavity is a function of the radius and can be adopted in the form [4]:

$$
E(r)=E_{0}\left[1+\left(k_{1}-1\right)\left(\frac{a}{r}\right)^{m}\right]
$$

where $E_{0}$ - is the modulus of elasticity in the virgin rock mass; $k_{1}=\frac{E_{a}}{E_{0}}$

$E_{a}$ - is the modulus of elasticity on the cavity countour, for $r=a$;

$m$ - is an empirical coefficient.

The value of the parameters $\mathrm{k}_{1}$ and $\mathrm{m}$ depends on the size of the disturbance zone and is determined experimentally [4]. The dimensions of the zone of artificial fracturing are directly dependent on the explosion power and in inverse relationship to the strength of the rocks being destroyed [4]. 


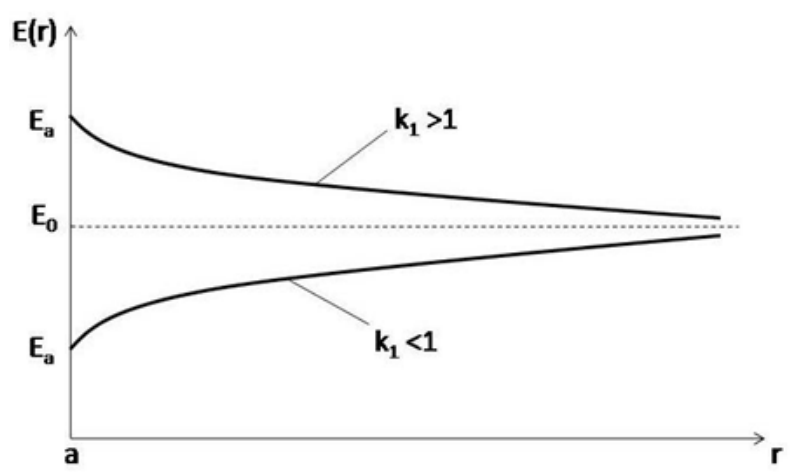

Fig. 1. Variation of rock mass modulus of elasticity near the cavity created by explosion.

\section{Methodology}

The ratio (1) properly describes experimental curves. Choosing the appropriate m, you can approximate a wide range of dependencies with both a slow and fast rate of change in the value of E, which corresponds to a larger or smaller area of the massif with changed properties.

Parameter $k_{1}>1$ in expression (1) corresponds to the case of rock compaction during explosion, and $k_{1}<1$ - to loosening (fracturing).

Expression (1) is successfully used by the authors of works devoted to solving problems with allowance for a one-dimensional inhomogeneity $[5,6]$.

When solving problems related to the determination of the stress-strain state of a rock mass under the influence of temperature effects, it becomes necessary to take into account the so-called indirect heterogeneity, while the construction of the dependence of the deformation characteristics (modulus of elasticity) on the coordinates consists of two stages.

First, the dependence of the modulus of elasticity on the temperature acting in the considered massif is determined. Thus, studies [7] of the character of the change in the Young's modulus in soils (clays) in the case of a uniaxial stress state at different temperatures have shown that with an increase in the heating temperature, this basic deformation characteristic of soils decreases (Fig. 2).

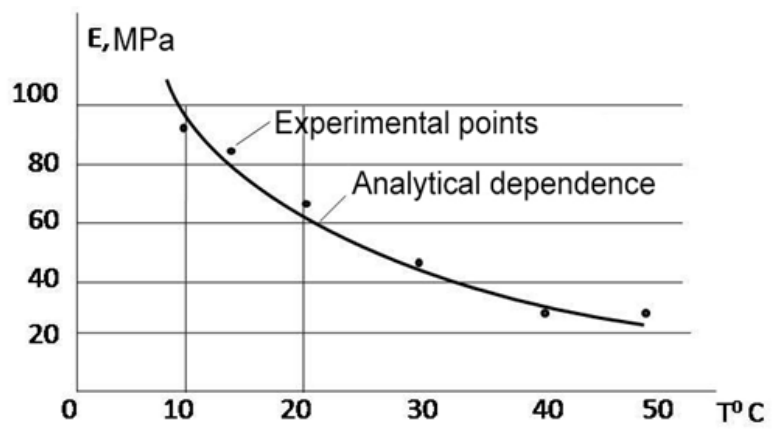

Fig. 2. Dependence of clay elasticity modulus on temperature.

Similar studies have also been carried out for rocks [2], but already for triaxial compression at much higher temperatures, since at relatively low temperatures the rocks (eg 
basalt) practically do not change their elastic properties (Fig. 3). Here, as in the first case, when the temperature rises, the modulus of elasticity decreases significantly (for example, in the granite, the Young's modulus at room temperature is almost three times larger than at $T=800^{\circ} \mathrm{C}$ ).

The results of the obtained experimental studies can be approximated with sufficient accuracy by means of a fairly simple functional dependence of the elasticity modulus of the material of the massif on temperature:

$$
E(T)=E_{0} \exp (-\delta T)
$$

Where $E_{0}$ - is the modulus of elasticity of the unheated massif; $T-$ is the heating temperature; - $\delta$ is the empirical coefficient.

Fig. 2 and Fig. 3 show approximating relationships of the type (2). It can be seen that in some cases, the coincidence with the experimental data is quite good throughout the temperature range (Fig. 2), and on some curves the function (2) makes it possible to describe the change in the modulus of elasticity as a function of temperature only on a part of the interval (Fig.3).

For superhard rocks of the basalt type, the relation (2) can be somewhat refined:

$$
\begin{array}{ll}
E(T)=E_{0} & a t T \leq 300^{\circ} \mathrm{C} \\
E(T)=E_{0} \exp (-\delta T) & \text { at } T>300^{\circ} \mathrm{C}
\end{array}
$$

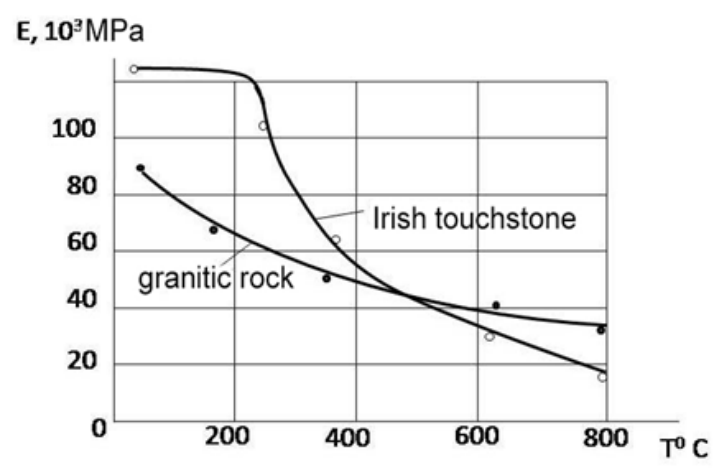

Fig. 3. Dependencies of solid rock elasticity modulus on temperature.

If the task related to the determination of temperature stresses considers a stationary heat flux, for example, caused by storage of products that tend to self-heating in the cavity (a constant temperature is maintained on the cavity surface), then the corresponding steadystate regime for a spherical cavity can be described by the dependence:

$$
T(r)=\frac{\left(T_{0}-T_{1}\right) a b}{r(b-a)}+\frac{T_{1} b-T_{0} a}{b-a}
$$

where $T_{0}$ - is the temperature on the contour of the cavity; $T_{1}$ - in the massif for heating; $a$ - is the radius of the cavity; $b>>$ a is the conditional radius of the outer contour of the massif. 


\section{Results}

Thus, in such tasks, taking relations (2) and (3) into account, it is easy to go from the E(T) dependence to $\mathrm{E}(\mathrm{T}(r))$ dependence.

If it is necessary to take into account both the temperature and the cracking of the rock, then the dependence of the modulus of elasticity can be written in the form [8]:

$$
E(T)=E_{0} \exp (-\delta T(r))\left[1+\left(k_{1}-1\right)\left(\frac{a}{r}\right)^{m}\right]
$$

\section{Conclusions}

One of the important issues that are considered in many studies on the calculations of inhomogeneous bodies is the question of the influence of the Poisson's ratio on the stressed state. Due to the lack of experimental data, this coefficient and the density of the material were considered to be constant in the majority of works, although, in principle, one can also assume their dependence on the coordinates of the points of the body.

\section{References}

1. F.D. Stacey, Physics of the Earth (Mir, Moscow, 1972)

2. G.A. Krupennikov, N.A. Filatov, Stress distribution in rock masses (Nedra, Moscow, 1972)

3. N.A. Tsytovich, Ground mechanics (Higher school, Moscow, 1979)

4. V.V. Rukin, K.V. Ruppeneit, Interaction mechanism of pressure tunnel lining with rock mass (Science, Moscow, 1969)

5. V.I. Andreev, Computational Methods and Experimental Measurements, 2, 202-216 (2014)

6. V.I. Andreev, A.S. Avershyev, Procedia Engineering, 111, 30-35 (2015)

7. S.R. Meschyan, Grounds mechanical properties and laboratory methods of their identification (Nedra, Moscow, 1974)

8. I.I. Frolova, Scientific review, 12, 94-99 (2015) 